\title{
Waray Scripting Language (WSL): A Mother Tongue-Based Scripting Language
}

\author{
Rolando Real Codilan \\ Instructor, College of Computer Studies \\ Eastern Samar State University, Borongan City Philippines \\ roland.r.codilan@gmail.com
}

\begin{abstract}
:
The objective of this study was to develop a waray scripting language to introduce computer programming using mother tongue-based scripting language to the beginners especially the first year college students enrolled in computer-related programs in Eastern Samar State University and to evaluate the waray scripting language in terms of its usability, readability, and writability. This study used racket programming language an open-source programming language to develop WSL, racket helps programmers develop and quickly deploy new language. WSL was subjected to software evaluation in terms of usability, readability and writability were based on (ISO 9126) Software Product Quality Metrics. This study used developmental-evaluative research design. The result shows that WSL a mother-tongue-based scripting language is of acceptable and is compliant to the ISO software standard with the variables obtained the average weighted mean rated as 3.5 Acceptable in general. For readability, it also obtained the average weighted mean of 3.6 Acceptable in general and for usability, it obtained the average weighted mean of 3.4 Acceptable in general and it also obtained the average weighted mean of 3.5 of all variables and was rated Acceptable in general. This implies that students have an optimistic response towards WSL. A modified syntax for better readability can also be introduced to enhance more the quality of the WSL.
\end{abstract}

\section{KEYWORDS:}

ISO 9126 , waray scripting language, mother-tongue based scripting.

\section{INTRODUCTION}

Eastern Samar State University in Borongan City is an educational institution with its primary goal is to produce excellent graduates in all fields of study offered in this university. Students enrolled in computer-related programs in this university are using scripting language especially the first year college students. It is a flexible language that provides fast program execution. This study would like to develop a scripting language in Waray. Waray is one of the major language spoken in the Philippines, mostly in the Eastern Visayas Region. In this study, WSL a mother-tongue-based scripting language is used to serve as a tool for first-year college students enrolled in a computer-related program in Learning Programming easily.

A scripting language is a programming language that employs a high-level construct to interpret and execute one command at a time. In general, scripting languages are easier to learn and faster to code more than structured and compiled languages such as $\mathrm{C}$ and $\mathrm{C}++$ Magaret Rouse, $2016^{4}$. There are lots of scripting languages that are available worldwide, this scripting language varies according to the purpose. Every scripting language has its own set of words (keywords) and syntaxes used that start a computer to perform certain tasks there are some scripting languages around the world that uses their own dialect as keywords for easy understanding of the locals.

Most of all, the popular scripting languages that are being used today are in the English language. There are articles that attempted to explain instructional language issues. Fischer and Perez, $2008^{1}$ said that students may struggle to learn the content in their second language if their academic knowledge is not sufficiently strong as their first language. That's why many people are having difficulties in learning scripting especially to those non-native speakers of English due to their lack of knowledge in the English language. This situation leads to the possible problems of understanding and remembering the keywords because of the language used by the scripting languages used in teaching programming on other countries. 
Programmers in the four corners of the world developed programming languages that could help learn to programming easier. They wrote programming languages using their own national languages or dialects. Leon Lukaszewics, $1961^{3}$ developed a Polish programming language called SAKO. According to Jim Cummins, 1991². To accomplish successful teaching and learning, teachers is required to use students' native language for instruction. The purpose of this study was to develop a Waray Scripting Language (WSL) a mother-tongue-based scripting language that will help and enhance the programming skills of the students in the university.

\section{READABILITY VS. WRITABILITY}

\subsection{Readability}

Readability simply means that the programming language is easy to read and understand by the programmers because the language construct is nearly the same as natural human language. This criterion was used as a gauge to evaluate the quality of WSL.

\subsection{Writability}

Writability means that it is easy and fast to create programs in that language because the language construct has minimal symbols which do not require many statements and focuses on simplification of code (concise).

Readability and Writability contradict each other, a readable programming language does not always mean it is writable (vice versa). Being a readable language means that you need to write extra or sometimes unnecessary code to make it more understandable, but on the other hand if the languages omit these codes ( to make writable easy and fast) programs will be obscure and the reader needs additional documentation to understand the code. Readability and writability should not be biased on the level of skills of the person who reads and writes the code. Non-programmers and beginners might find it difficult to read and write on that particular programming language, but experienced programmers on that language will find it easy. Readability and writability should be based on syntax (language constructs) and semantics meaning and how it is understood. (https://williamarchibaldspooner.wordpress.com)

\subsection{Code Readability}

In the study of Buse \& Weimer, 2010 , Code Readability can be defined as a human judgment on how easy it is to understand a program source code. Furthermore, by Yahya Tashtous and Izzat Alsmadi, $2013^{6}$, although the readable code is less erroneous, more reusable and code readability is not easy to measure by a deterministic function same as maintainability, reliability, and reusability. However, a source code features that affect readers' understanding of existing source code. Studying the impact of programming Features on Code Readability.

\section{METHODOLOGY}

This chapter covers the methods and steps used in developing this scripting language. This part also discusses the development model used in this study.

\subsection{Software Development}

In developing the scripting language, this study used racket programming language because racket was designed to create other programming languages. Racket helps programmers create and quickly deploy new languages.

In designing the syntax, this study consulted a linguist on what appropriate Waray words to be used as keywords in WSL (Waray Scripting Language).

\subsection{Software Development Model}

This study used prototyping model in the development of the Waray scripting language. The prototyping Model is a systems development method (SDM) in which a prototype (an early approximation of a final system or product) is built, tested, and then reworked as necessary until an acceptable prototype is finally achieved from which the complete system or product can now be developed. This model works best in scenarios where not all of the project requirements are known in detail ahead of time. It is an iterative, trial-and-error process that takes place between the developers and the users.

The Prototype Model was used in developing this programming language to achieve he requirements and to meet the objectives of this study by trial and error process until a good prototype is achieved. 


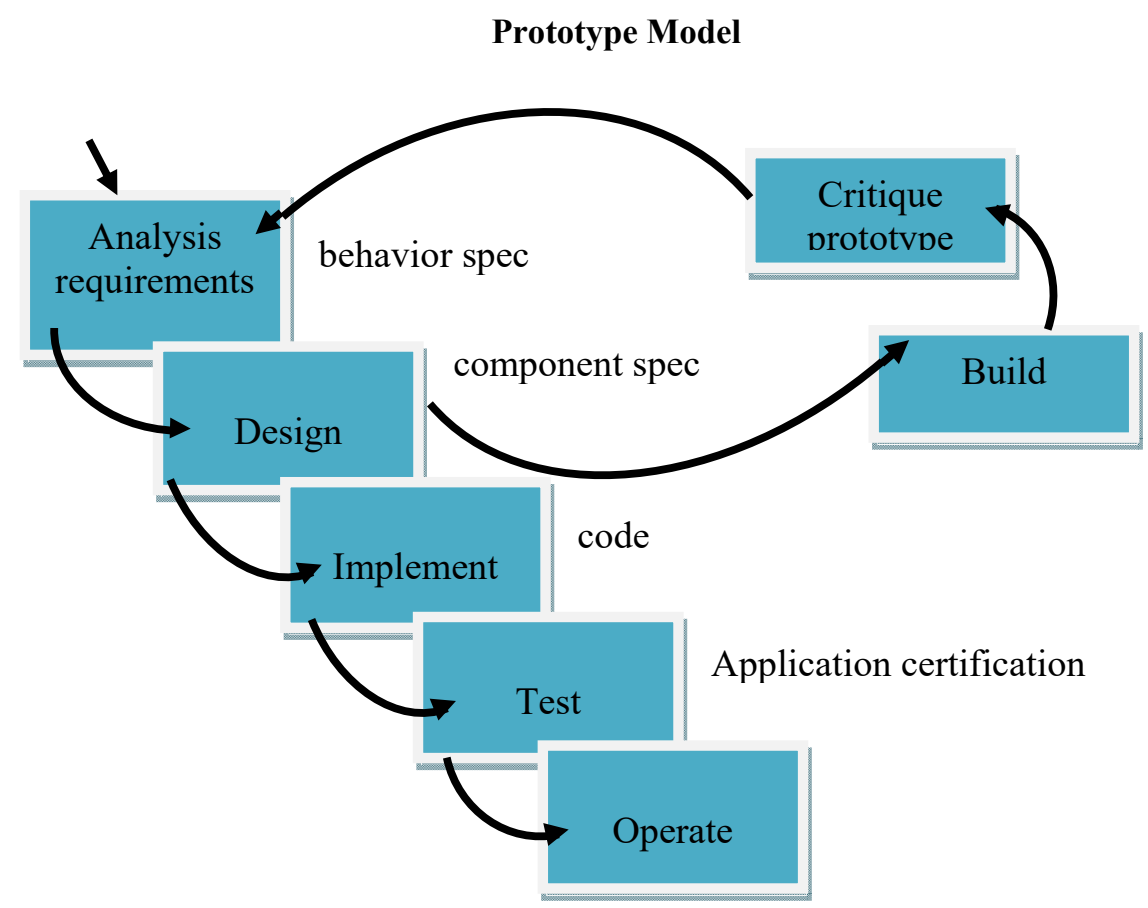

Figure 1. Prototyping Model

\subsection{Software Employed}

WSL was developed using Racket programming language. This language is free and open-source that were designed to develop other programming languages.

\subsection{Hardware Employed}

The following hardware specification is the minimum requirement to run and use WSL successfully:

- Processor Intel(R) Core(TM)2 Duo CPU $1.67 \mathrm{GHz}$

- $\quad$ Ram 2.00 GB

- Hard Disk 500 GB

- Display 1280x800 (32bit) $(60 \mathrm{~Hz})$

\subsection{WSL KEYWORDS AND SYNTAXES}

Table 1 shows the list of keywords of WSL including the syntaxes employed per keyword. These syntaxes served as a guide for programmers in using WSL and running an error-free program.

Table 1. Keywords and Syntaxes used in WSL

\begin{tabular}{|c|c|c|}
\hline Keywords & Description & Syntax \\
\hline Isurat-an & Displays strings or integers & (Isurat-an $s t x)$ \\
\hline Ideklara-an & Declares a variable & $\begin{array}{l}(\text { Ideklara-an }<\mathrm{id}><\text { expr }>) \\
(\text { Ideklara-an }(<\mathrm{id}><\mathrm{id}>*)<\text { expr }>+)\end{array}$ \\
\hline Dugangi-an & Add numbers & $($ dugangi-an $<$ num $>$ hin $<$ num $>*$ ) \\
\hline Ibani-an & Subtract numbers & (ibani-an $<$ num $>$ hin $<$ num $>*$ ) \\
\hline Dobleha-an & Multiply numbers & (dobleha-an $<$ num $>$ hin $<$ num $>*)$ \\
\hline Tungaa-an & Divide numbers & (Tungaa-an $<$ num $>$ hin $<$ num $>$ ) \\
\hline Mamadako-an & Greater than & (Mamadako-an $<$ num $>$ hin $<$ num $>$ ) \\
\hline Mamaguti-an & Less than & (Mamaguti-an $<$ num $>$ hit $<$ num $>$ ) \\
\hline Maadako-o-parehas-an & Greater than or equal & (Mamadako-o-parehas-an $<$ num $>$ hit $<$ num $>$ ) \\
\hline Diri-parehas-an & Not Equal & (Diri-parehas-an $<$ num $>$ hit $<$ num $>$ ) \\
\hline Mamaguti-o-parehas-an & Less than or equal & (Mamaguti-o-parehas-an $<$ num $>$ hit $<$ num $>$ ) \\
\hline Kun...Kun-diri & If...else & $\begin{array}{l}\text { (kun }(<\text { condition }>) \text { true-expr kun-diri false- } \\
\text { expr) }\end{array}$ \\
\hline
\end{tabular}


Figure 2. shows the process of code flow for WSL during runtime including their explanation.

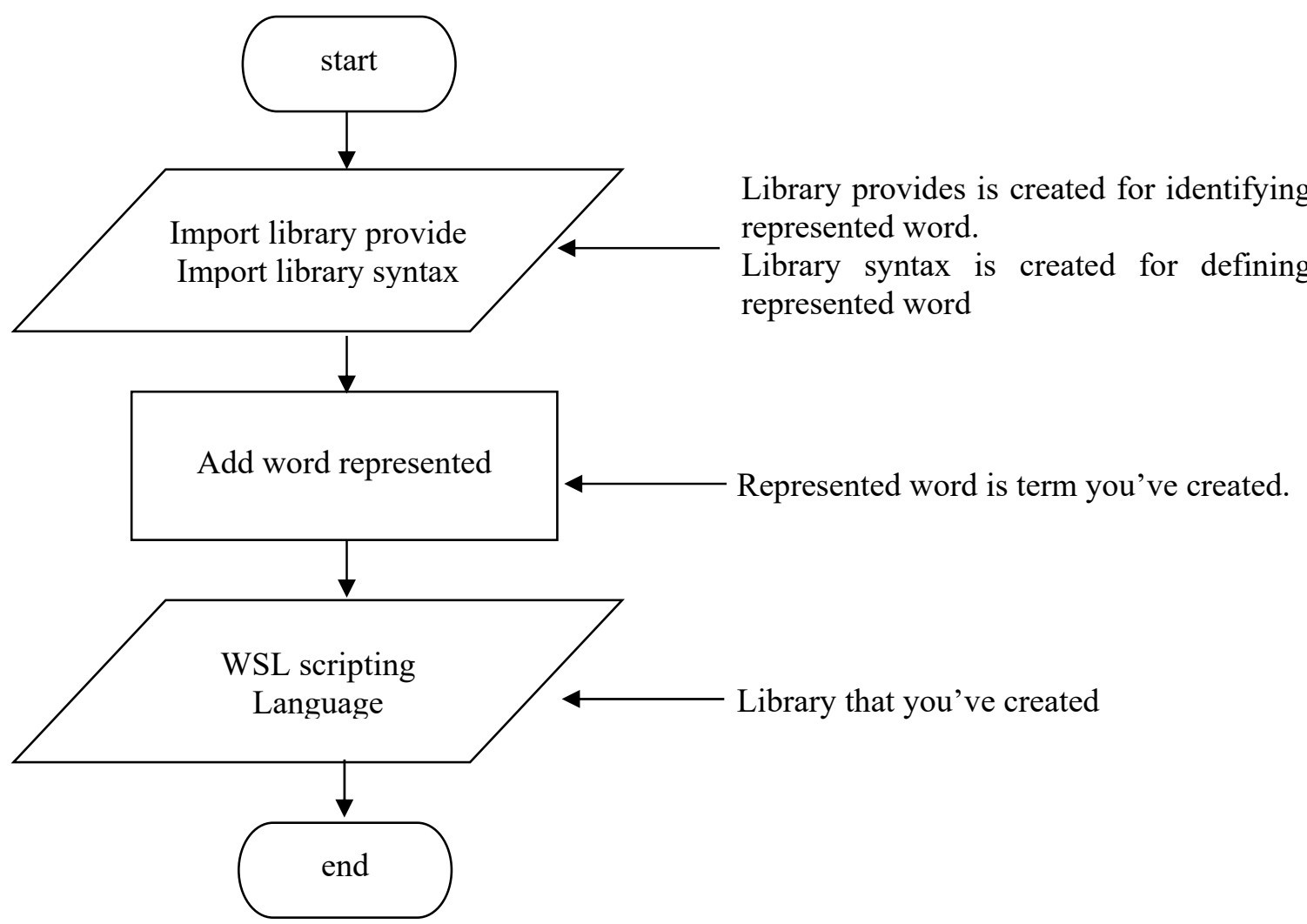

Figure 2. The basic code flow for (WSL) during runtime.

\section{Evaluation}

\section{Research Design}

This study used a developmental-evaluative research design in which the focus was more on the impact of the WSL as a tool for the first year college students in Eastern Samar State University in their learning in programming subject using scripting language. WSL was subjected for quality evaluation in terms of (1) Usability, (2) Readability, and (3) Writability.

\section{Data Gathering and Instrumentation}

This study used a questionnaire in collecting data from the respondents of the study. The questionnaire contained two levels of questions the first level is all about software development. The last level of question consists of three factors that respond to the performance of the study these are usability, readability, and writability. Each factor has four elements and the respondents were instructed on how to answer each level of questions. Each question was rated the following Likert Scale: (5) Strongly Agree, (4) Agree, (3) Neither agree or disagree, (2) Disagree, and (1) Strongly disagree.

\section{Data Analysis}

This study used questionnaires to obtain data and information from the respondents concerning the performance of WSL in terms of usability, readability, and writability. Questionnaires responses were answered. Tabulated and analyzed using Frequency and Weighted Mean. Tabulated data were interpreted using table 3 below.

Table 3. Interpreted Scale

\begin{tabular}{|c|c|}
\hline Scale & Interpretation \\
\hline $4.20-5.0$ & Strongly Acceptable \\
\hline $3.40-4.10$ & Acceptable \\
\hline $2.60-3.30$ & Neither Acceptable nor unacceptable \\
\hline $1.80-2.50$ & Unacceptable \\
\hline $1.00-1.70$ & Strongly unacceptable \\
\hline
\end{tabular}




\subsection{RESULT AND DISCUSSION}

This Chapter shows the result of the development process and the result of the evaluation of the quality and usability of WSL.

\section{Software Development}

\section{Waray Scripting Language (WSL) User Interface}

WSL was designed using Rackect, an open-source programming language that is intended to design new other languages. This study created a library under Racket and imported this to enable the execution of WSL codes in DrRacket interface. Figure 4 shows the DrRacket interface with library "\#lang WSL". This "\#lang WSL" should be the first line of WSL code to allow end-users to run the compiled scripting.

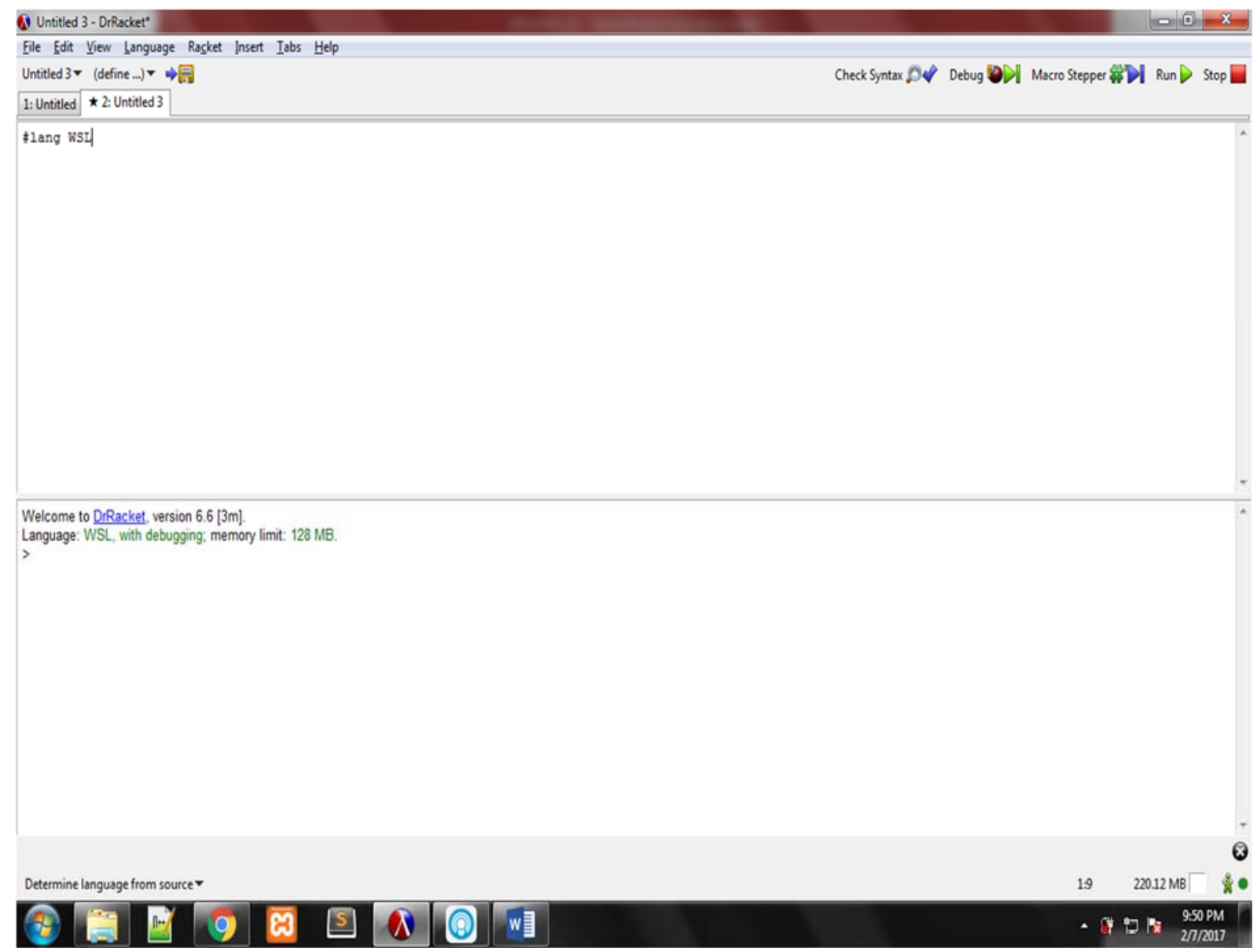

Figure 4. DrRacket - WSL User Interface 
The user will type WSL codes in the text area and click the run button on the upper-right corner to execute the program. The output of the program is seen in the output box below the screen. Figure 5 shows the WSL code with a running program.

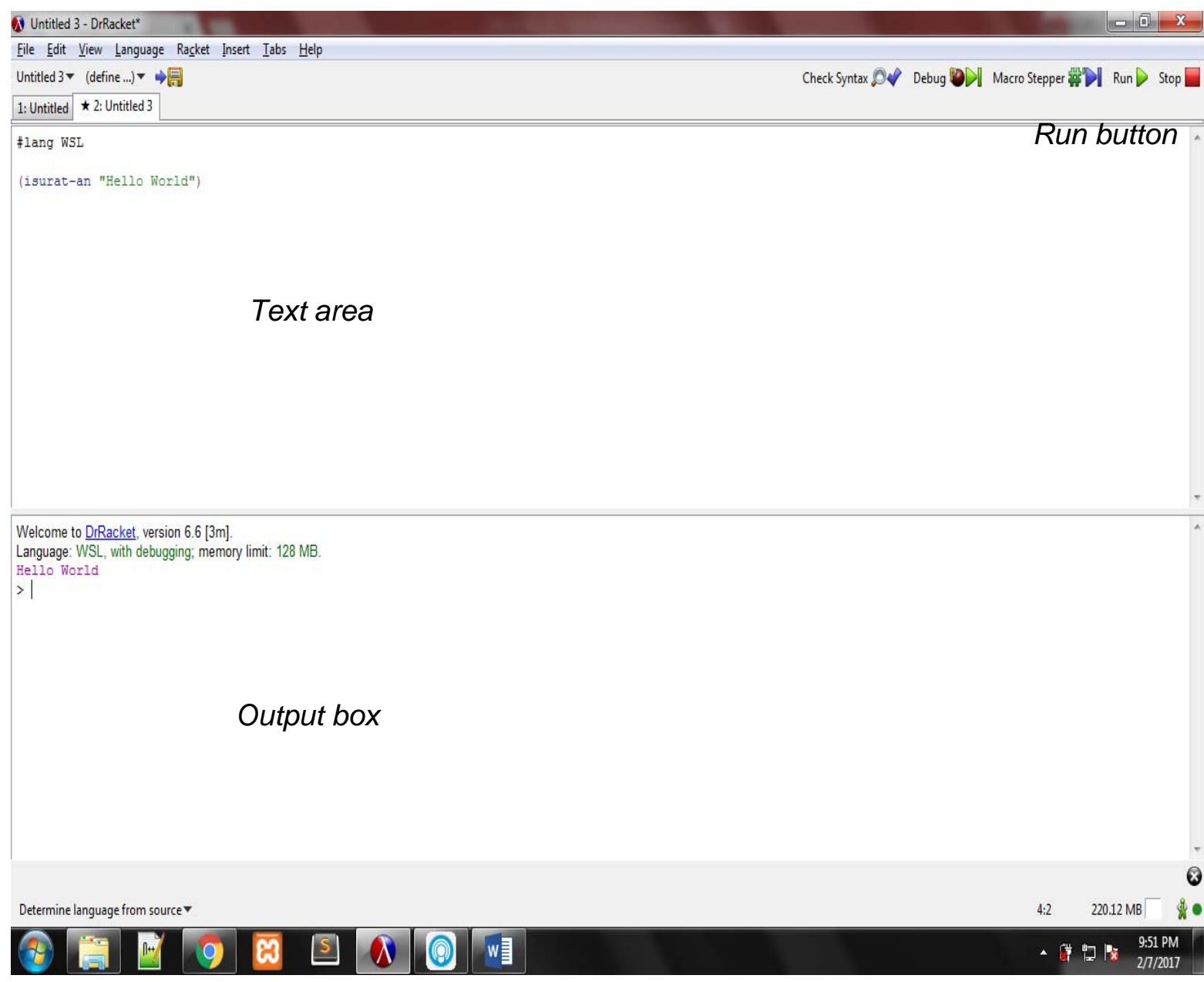

Figure 5. Code with Running Syntax

\section{Result}

The data from the evaluation were calculated and analyzed through the use of appropriate statistical techniques. 141 respondents were made to answer the questionnaire to verify the quality of WSL in terms of usability, readability, and writability.

Table 4. Performance Level of the Proposed WSL: Waray Scripting Language in terms of Usability.

\begin{tabular}{|c|c|c|c|c|c|c|c|}
\hline CRITERIA & \multicolumn{5}{|c|}{ Responses (f) } & \multirow{2}{*}{$\begin{array}{c}(\mathrm{N}=141) \\
\text { Weighted } \\
\text { mean }\end{array}$} & \multirow{2}{*}{ Interpretation } \\
\hline Usability & 1 & 2 & 3 & 4 & 5 & & \\
\hline a. Performance & 0 & 0 & & & & 3.5 & Acceptable \\
\hline b. Solution & 0 & 0 & & & & 3.5 & Acceptable \\
\hline c. Actual output & 0 & 0 & & & 0 & 3.3 & Neutral \\
\hline Average Weighted Mean & & & & & & 3.4 & Acceptable \\
\hline
\end{tabular}

Table 4 shows the result of the evaluation in terms of usability. The respondents gave 3.5 or acceptable rating under performance, 3.5 or acceptable for the solution and 3.3 or neither acceptable nor unacceptable for the actual output. Having an average rate of 3.4 interpreted as acceptable. 
Table 5. Performance Level of the Proposed WSL: Waray Scripting Language in terms of Readability.

\begin{tabular}{|l|c|c|c|c|c|c|c|}
\hline \multicolumn{7}{|c|}{ CRITERIA } & \multicolumn{7}{c|}{ Responses (f) } & $\begin{array}{c}\text { (N=141) } \\
\text { Weighted } \\
\text { mean }\end{array}$ & Interpretation \\
\hline Readability & 1 & 2 & 3 & 4 & 5 & & \\
\hline a. Performance & 0 & 2 & & & & 3.7 & Acceptable \\
\hline b. Solution & 0 & 3 & & & & 3.4 & Acceptable \\
\hline c. Actual output & 0 & 2 & & & & 3.7 & Acceptable \\
\hline Average Weighted Mean & \multicolumn{70}{|l|}{} & & 3.6 & Acceptable \\
\hline
\end{tabular}

Table 5 shows the performance of the proposed study under the criterion Readability. The respondents gave 3.7 acceptable under performance, 3.4 acceptable for the solution and 3.7 acceptable for the actual output; with an average result of 3.6 interpreted as acceptable indicates that more than a half of respondents agreed that WSL conforms to its readable quality.

Table 6. Performance Level of the Proposed WSL: Waray Scripting Language in terms of Writability.

\begin{tabular}{|c|c|c|c|c|c|c|c|}
\hline CRITERIA & \multicolumn{5}{|c|}{ Responses (f) } & \multirow{2}{*}{$\begin{array}{c}(\mathrm{N}=141) \\
\text { Weighted } \\
\text { mean }\end{array}$} & \multirow{2}{*}{ Interpretation } \\
\hline Writability & 1 & 2 & 3 & 4 & 5 & & \\
\hline a. Performance & 0 & 2 & & & & 3.4 & Acceptable \\
\hline b. Solution & 0 & 3 & & & & 3.6 & Acceptable \\
\hline c. Actual output & 0 & 2 & & & & 3.6 & Acceptable \\
\hline Average Weighted Mean & & & & & & 3.5 & Acceptable \\
\hline
\end{tabular}

Table 6 shows the performance of the Proposed study under the criterion Writability. The respondents gave 3.4 acceptable under performance, 3.6 acceptable for the solution and 3.6 acceptable for the actual output. WSL writability quality earned $n$ average weighted mean of 3.5 interpreted as acceptable. The result indicates that WSL conforms to the writability standard of programming languages.

Table 7. Summary of Performance of the Proposed WSL: Waray Scripting Language

\begin{tabular}{|c|c|c|}
\hline CRITERIA & Weighted Mean & Interpretation \\
\hline Usability & 3.4 & Acceptable \\
\hline Readability & 3.6 & Acceptable \\
\hline Writability & 3.5 & Acceptable \\
\hline Overall Weighted Mean & 3.5 & Acceptable \\
\hline
\end{tabular}

Table 7 shows the summary of WSL: Waray Scripting Language quality evaluation results. The Language earned an overall weighted mean of 3.5 interpreted as acceptable. This overall result indicates that WSL adheres to the quality of a scripting language.

This result indicates that WSL is of usable quality and can be used for learning programming and creating programming applications.

\section{SUMMARY}

\section{Summary}

This study was conducted to develop a scripting language that uses waray dialect as keywords and evaluate its performance. The purpose of developing WSL or Waray Scripting Language is to introduce programming to beginners here in Eastern Samar State University. WSL or Waray Scripting Language can only construct basic codes because it is designed for a beginner which is first-year college students in Eastern Samar. The syntax of WSL ( Waray Scripting Language) is much more different than C programming language, the syntax of WSL is parenthesized and verbose unlike C and other programming languages that uses symbols. 


\section{References:}

[1] Fischer and Perez, 2008. Understanding English Through Mathematics

[2] Jim Cummins, 1991. "A Conceptual framework of Bilingual Special Education Teacher Program".

[3] Leon Łukaszewicz, 1961. "System automatycznego kodowania SAKO"

[4] Magaret Rouse, 2001. What scripting languages should every DevOp know?

[5] Raymond P.L. Buse \& Westley Weimer, 2010. Learning a Metric for Code Readability.

[6] Yahya Tashtous and Izzat Alsmadi, 2013. Impact of Programming Features on Code Readability

\section{APPENDIX A}

(WSL CODE)

\section{\#lang racket}

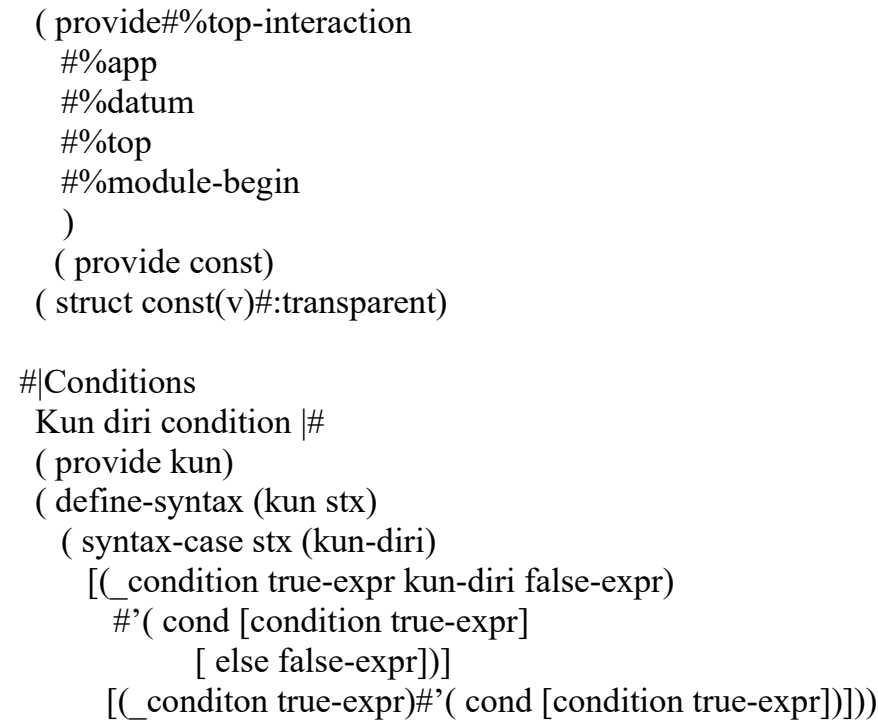

\#| Print: Displays strings or integers

Isurat-an \#

(provide isurat-an)

( define-syntax isurat-an

( syntax-rule ()

$[($ isurat stx $)$ ( printf stx $)]$

$[($ isurat (void) $)(\operatorname{printf}($ void $))]$

$[($ isurat stx 1 stx $2 \ldots)($ printf stx 1 stx $2 \ldots)]))$

\#| Print: Displays strings or integers next line

Isurat-in $\mid \#$

( provide isurat-in)

( define-syntax isurat-in

( syntax-rules ()

[( isurat stx)( displayin (stx)]

[( isurat (void))(displayin (void)) ]

$[($ isurat stx 1 stx $2 \ldots)($ displayin stx 1 stx $2 \ldots)]))$

\#| Declares Variable/Identifiers

Ideklara-an |\#

( provide ideklara-an)

( define-syntax ideklara-an

( syntax-rules ()

[( ideklara-an id expr)(define id expr)]

[( ideklara-an (id1 id2...) expr...)( define (id1 id2...) expr...)]))

\#| Operators:

Add $=$ dugangi-an 


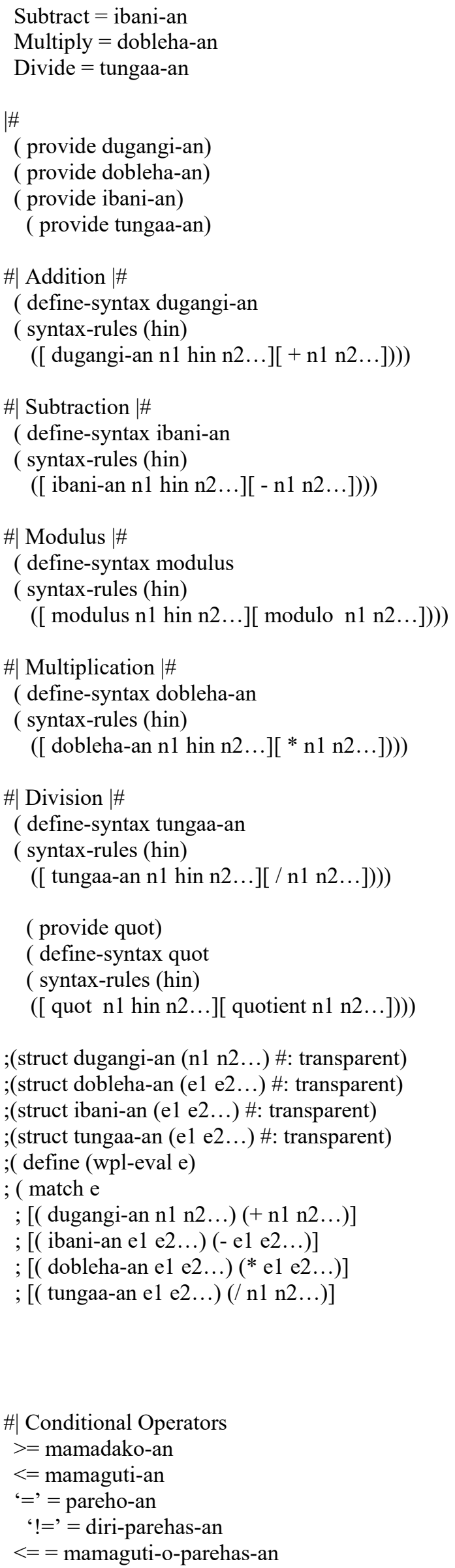




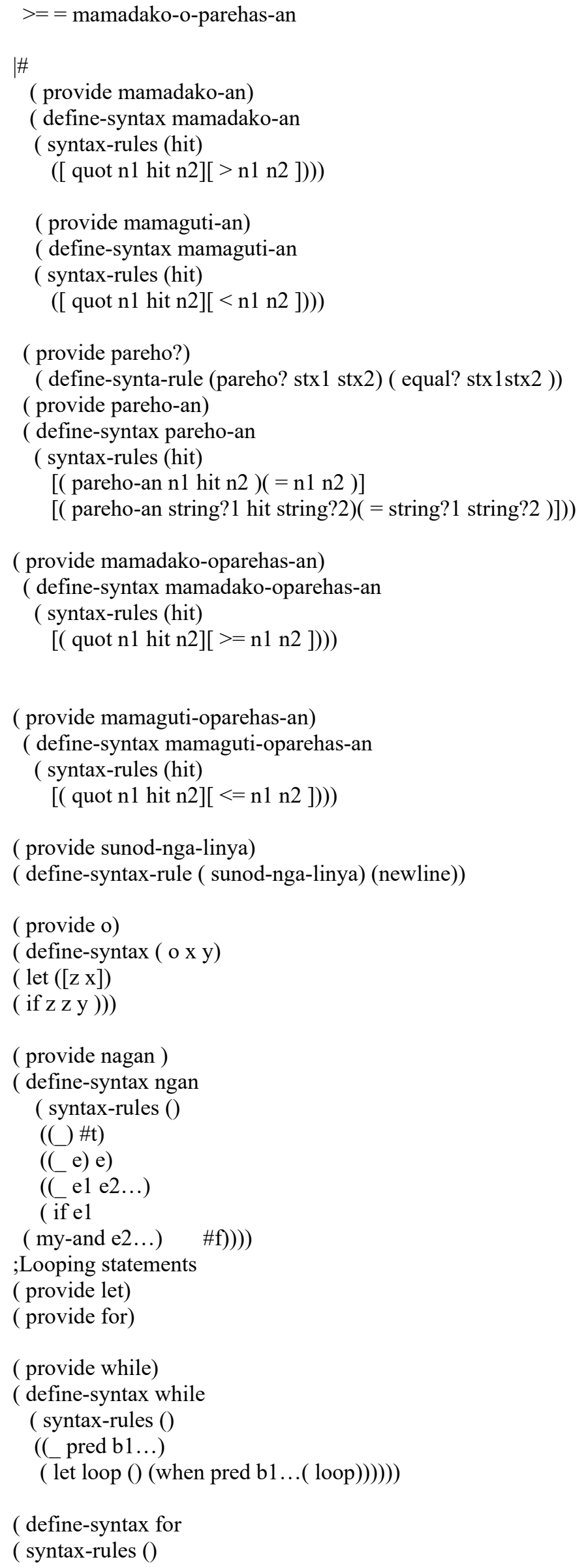




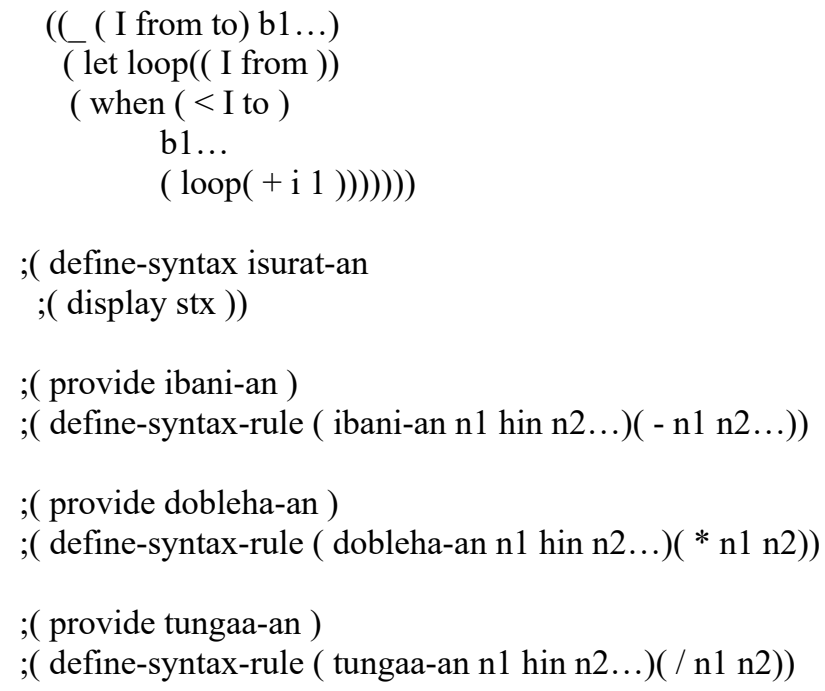




\section{APPENDIX B}

\section{WSL SAMPLE PROGRAM CODES}

\section{Leap Year}

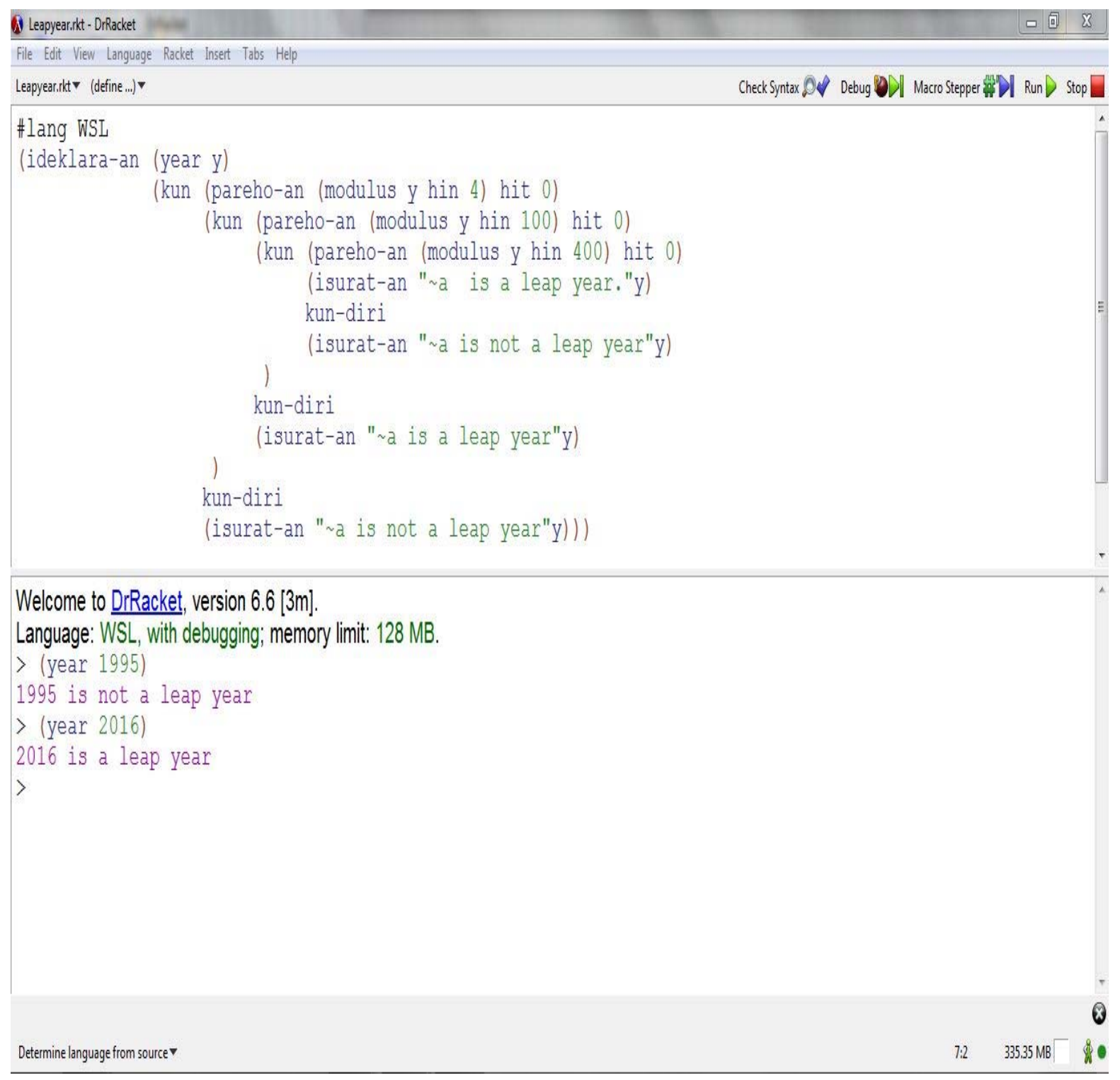




\section{Multiplication Table}

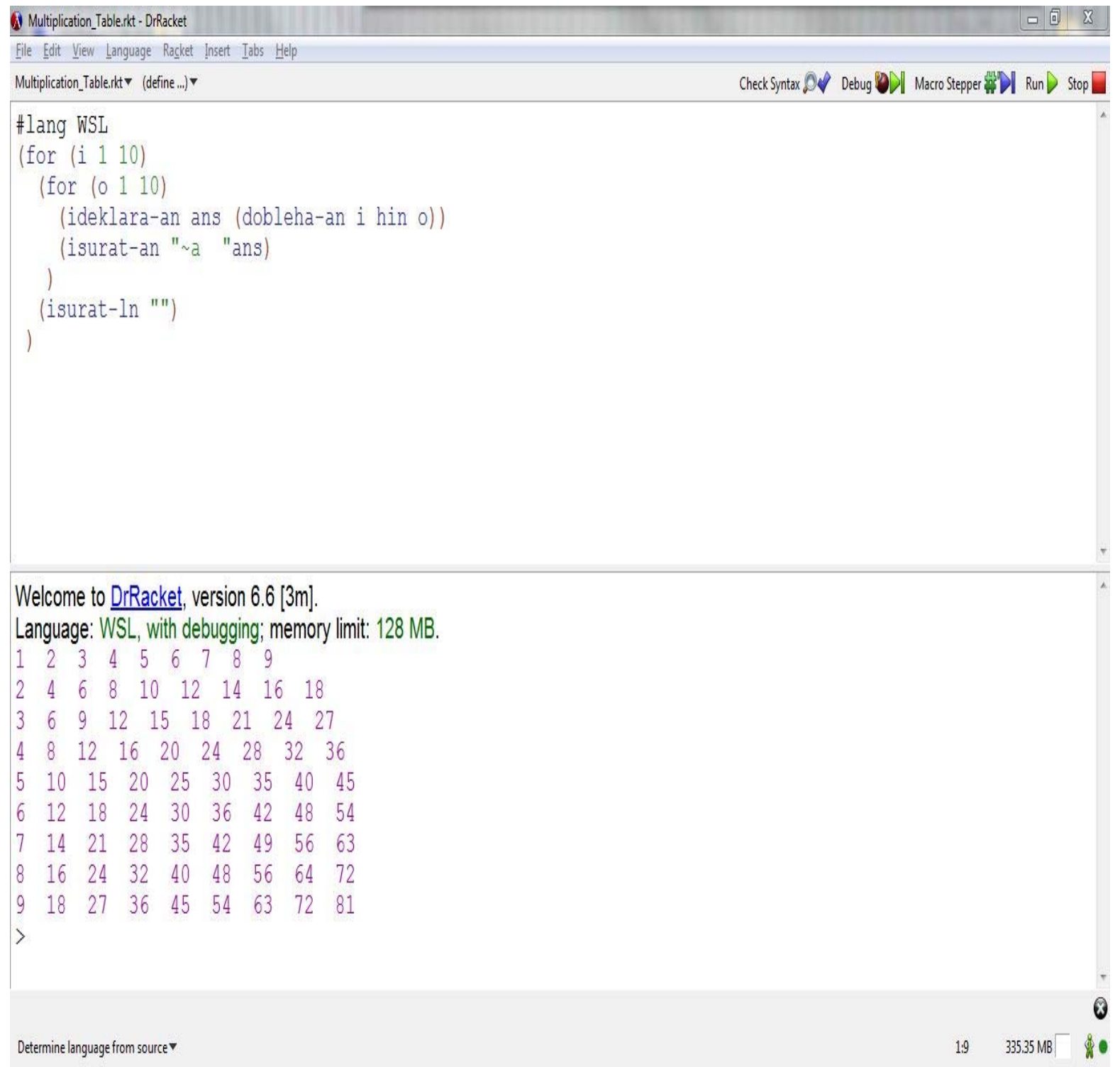




\section{Odd Even Number}

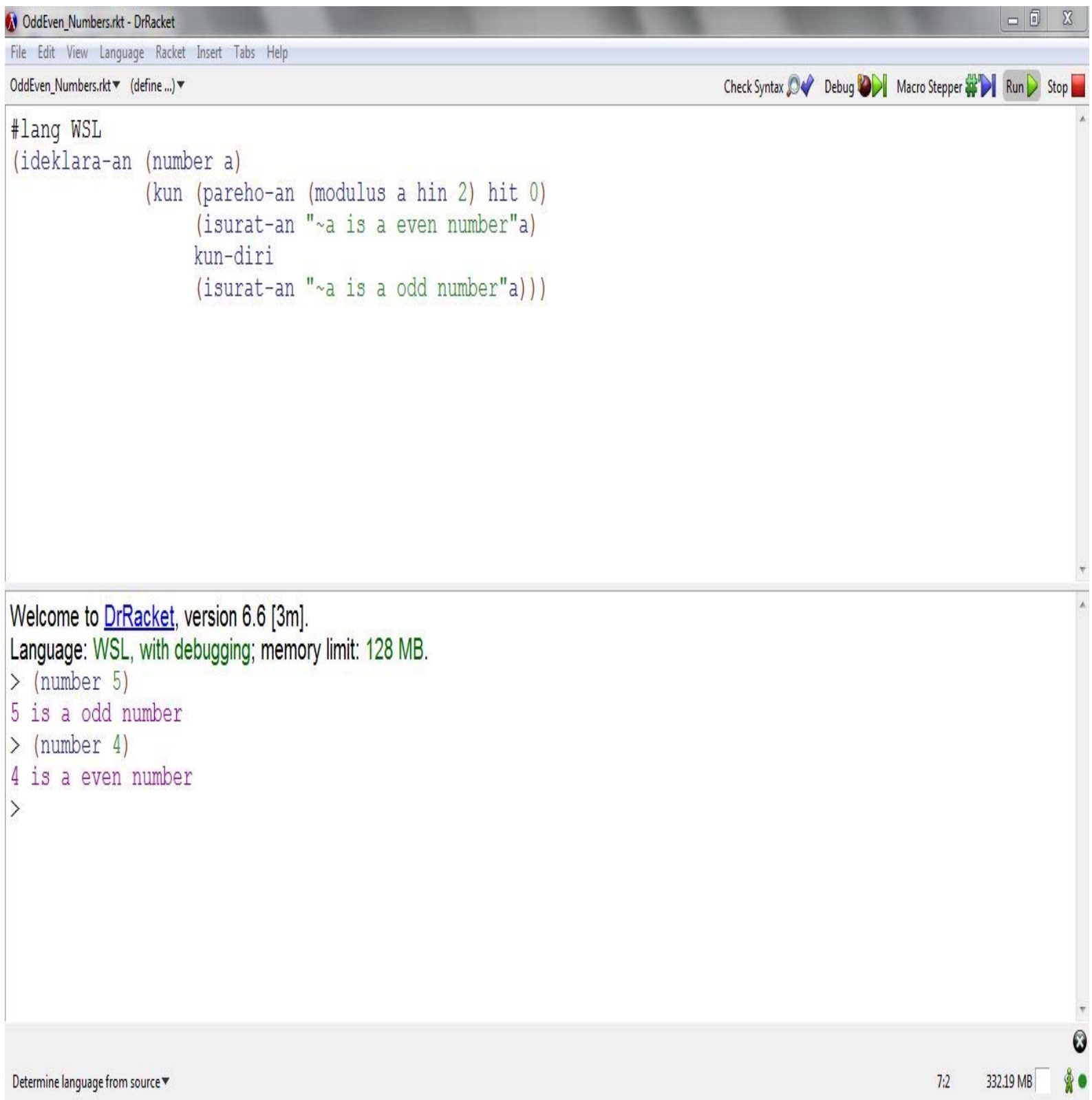




\section{Pyramid}

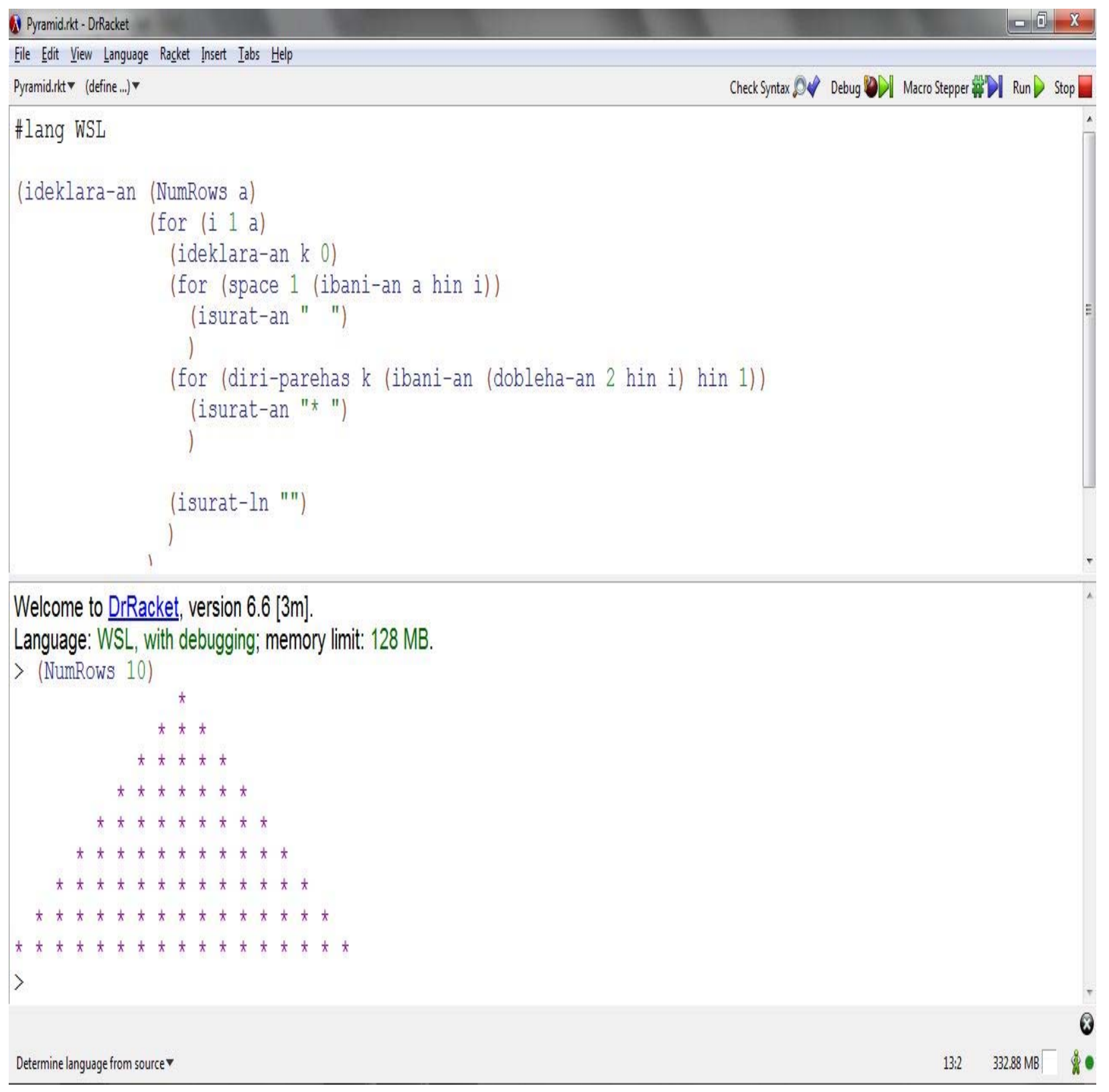




\section{Temperature}

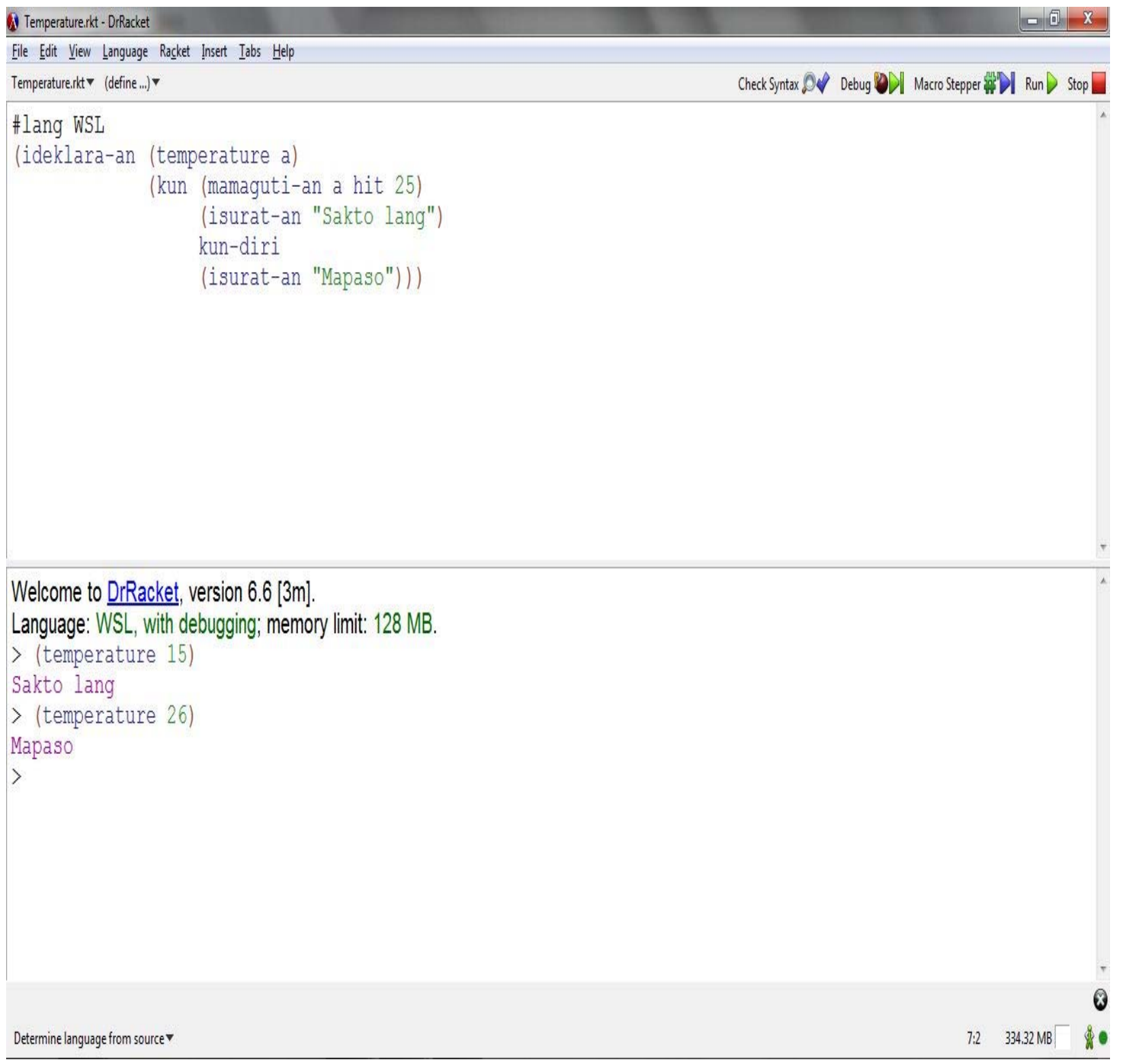

